SLAC-PUB-8833

BABAR-PROC-01/24

hep-ex/0105057

May, 2001

\title{
Measurements of $\mathrm{CP}$ violation, mixing and lifetimes in $B$ meson decays with the $B_{A} B_{A R}$ experiment at PEP-II.
}

\author{
Riccardo Faccini \\ University of California San Diego and Università "La Sapienza" Roma \\ (for the BABAR Collaboration)
}

\begin{abstract}
The BABAR detector, which operates at the SLAC PEP-II asymmetric $e^{+} e^{-}$collider at energies near the $\Upsilon(4 S)$ resonance has collected about $23 \mathrm{M} B \bar{B}$ pairs in year 2000. Based on this data sample, we present a first study of $\sin 2 \beta$, with samples of $B^{0} \rightarrow J / \psi K_{S}^{0}, B^{0} \rightarrow \psi(2 S) K_{S}^{0}$ and $B^{0} \rightarrow J / \psi K_{L}^{0}$ decays. The measured value is $\sin 2 \beta=0.34 \pm 0.20$ (stat) \pm 0.05 (syst). In addition, we present preliminary measurements of charged and neutral $B$ meson lifetimes and $B^{0} \bar{B}^{0}$ oscillation frequency.
\end{abstract}

Contributed to the Proceedings of the 36th Rencontres de Moriond on Weak Interactions 10-17 Mar 2001, Les Arcs, France

Stanford Linear Accelerator Center, Stanford University, Stanford, CA 94309 


\section{Introduction}

The primary goal of the BABAR experiment at PEP-II is to over-constrain the Unitarity Triangle. The sides of this triangle can be measured through non- $C P$ violating physics, such as $V_{u b}, V_{c b}, V_{t d}$ measurements, while its angles are accessible through $C P$ violating processes ${ }^{1}$.

\section{PEP-II}

The PEP-II $B$ Factory ${ }^{2}$ is an $e^{+} e^{-}$colliding beam storage ring complex on the SLAC site designed to produce a luminosity of at least $3 \times 10^{33} \mathrm{~cm}^{-2} \mathrm{~S}^{-1}$ at a center-of-mass energy of $10.58 \mathrm{GeV}$, the mass of the $\Upsilon(4 S)$ resonance. In the 2000 run, the achieved average luminosity was $3.3 \times 10^{33} \mathrm{~cm}^{-2} \mathrm{~s}^{-1}$. The total collected luminosity was about $23 \mathrm{fb}^{-1}$. The machine is asymmetric with a High Energy Ring (HER) for the $9.0 \mathrm{GeV}$ electron beam and a Low Energy Ring (LER) for the $3.1 \mathrm{GeV}$ positron beam. This corresponds to $\beta \gamma=0.56$ and makes it possible to measure time dependent $C P$ violating asymmetries. It corresponds to an average separation of $\beta \gamma \mathrm{c} \tau=250 \mu \mathrm{m}$ between the two $B$ mesons vertices.

\section{$3 B A B A R$}

\subsection{Detector description}

The BABAR detector is described in ${ }^{3}$. The volume within the BABAR superconducting solenoid, which produces a $1.5 \mathrm{~T}$ axial magnetic field, consists of: a five layer silicon strip vertex detector (SVT), a central drift chamber (DCH), a quartz-bar Cherenkov radiation detector (DIRC) and a CsI crystal electromagnetic calorimeter (EMC). Two layers of cylindrical resistive plate counters (RPCs) are located between the barrel calorimeter and the magnet cryostat. All the detectors located inside the magnet have full acceptance in azimuth. The integrated flux return (IFR) outside the cryostat is composed of 18 layers of steel, which successively increase in thickness away from the interaction point, and are instrumented with 19 layers of planar RPCs in the barrel and 18 in the endcaps.

\subsection{Event reconstruction}

Charged particles are detected and their momentum is measured by a combination of the DCH and SVT. The charged particle momentum resolution is approximately given by $\left(\delta p_{T} / p_{T}\right)^{2}=$ $\left(0.0015 p_{T}\right)^{2}+(0.005)^{2}$, where $p_{T}$ is in $\mathrm{GeV} / c$. The SVT, with a typical resolution of $10 \mu \mathrm{m}$ per hit, provides excellent vertex resolution both in the transverse plane and in $z$. The vertex resolution in $z$ is typically $50 \mu \mathrm{m}$ for a fully reconstructed $B$ meson and of order $100 \mu \mathrm{m}$ for the distance among the two $B$ mesons when only one is fully reconstructed. Leptons and hadrons are identified using a combination of measurements from all the BABAR components, including the energy loss $\mathrm{d} E / \mathrm{d} x$ in the helium-based gas of the DCH (40 samples maximum) and in the silicon of the SVT (5 samples maximum). Electrons and photons are identified in the barrel and the forward regions by the EMC, and muons are identified in the IFR. In the barrel region the DIRC provides excellent kaon identification over the full momentum range above $250 \mathrm{MeV} / \mathrm{c}$. 


\section{$4 \sin 2 \beta$ measurement}

In $e^{+} e^{-}$storage rings operating at the $\Upsilon(4 S)$ resonance a $B^{0} \bar{B}^{0}$ pair produced in a $\Upsilon(4 S)$ decay evolves in a coherent $P$-wave until one of the $B$ mesons decays. If one of the $B$ mesons $\left(B_{\operatorname{tag}}\right)$ can be ascertained to decay to a state of known flavor at a certain time $t_{\text {tag }}$, the other $B\left(B_{C P}\right)$ is at that time known to be of the opposite flavor. For the measurement of $\sin 2 \beta, B_{C P}$ is fully reconstructed in a $C P$ eigenstate $\left(J / \psi K_{S}^{0}, \psi(2 S) K_{S}^{0}\right.$ or $\left.J / \psi K_{L}^{0}\right)$. By measuring the proper time interval $\Delta t=t_{C P}-t_{t a g}$ from the $B_{t a g}$ decay time to the decay of the $B_{C P}\left(t_{C P}\right)$, it is possible to determine the time evolution of the initially pure $B^{0}$ or $\bar{B}^{0}$ state:

$$
f_{ \pm}\left(\Delta t ; \Gamma, \Delta m_{d}, \mathcal{D} \sin 2 \beta\right)=\frac{1}{4} \Gamma \mathrm{e}^{-\Gamma|\Delta t|}\left[1 \mp \mathcal{D} \eta_{C P} \sin 2 \beta \times \sin \Delta m_{d} \Delta t\right]
$$

where the + or - sign indicates whether the $B_{\text {tag }}$ is tagged as a $B^{0}$ or a $\bar{B}^{0}$, respectively. The dilution factor $\mathcal{D}$ is given by $\mathcal{D}=1-2 w$, where $w$ is the mistag fraction, i.e., the probability that the flavor of the tagging $B$ is identified incorrectly. $\eta_{C P}$ is the $C P$ eigenstate of the final state and it is $\eta_{C P}=-1$ for the $J / \psi K_{S}^{0}$ and $\psi(2 S) K_{S}^{0}$ modes, $\eta_{C P}=+1$ for the $J / \psi K_{L}^{0}$ mode. Although less pure, the $J / \psi K_{L}^{0}$ mode is very important because the oscillation is expected to be opposite to the other ones. A direct $C P$ violation term proportional to $\cos \Delta m_{d} \Delta t$ could arise from the interference between two decay mechanisms with different weak phases. In the Standard Model, we consider that the dominant diagrams for the decay modes have no relative weak phase, so no such term is expected.

To account for the finite resolution of the detector, the time-dependent distributions $f_{ \pm}$for $B^{0}$ and $\bar{B}^{0}$ tagged events (Eq. 1) must be convoluted with a time resolution function $\mathcal{R}(\Delta t ; \hat{a})$ :

$$
\mathcal{F}_{ \pm}\left(\Delta t ; \Gamma, \Delta m_{d}, \mathcal{D} \eta_{C P} \sin 2 \beta, \hat{a}\right)=f_{ \pm}\left(\Delta t ; \Gamma, \Delta m_{d}, \mathcal{D} \eta_{C P} \sin 2 \beta\right) \otimes \mathcal{R}(\Delta t ; \hat{a})
$$

where $\hat{a}$ represents the set of parameters that describe the resolution function.

Finally, the time-dependent distributions need to account for the background with additional parameters that characterize both its sample composition and the $\Delta t$ distribution. The $\eta_{C P}=-1$ background components are parametrized with an ARGUS function and a small peaking component in the energy substituted mass $m_{\mathrm{ES}}$. While the ARGUS component is extracted from data, the peaking one is derived from data. The $\eta_{C P}=+1$ background is made of a component from $B$ decays in $J / \psi$, whose parameters are taken from $\mathrm{MC}$, and the other sources which are characterized using the $J / \psi$ mass sidebands.

Since no time-integrated $C P$ asymmetry effect is expected, an analysis of the time-dependent asymmetry is necessary.

\subsection{Analysis}

For this analysis, published in ${ }^{4}$, we use a sample of $23 \mathrm{fb}^{-1}$ of data recorded in year 2000, of which $2.6 \mathrm{fb}^{-1}$ was recorded $40 \mathrm{MeV}$ below the $\Upsilon(4 S)$ resonance (off-resonance data).

The measurement of the $C P$-violating asymmetry has five main components :

- Selection of the signal $B^{0} / \bar{B}^{0} \rightarrow J / \psi K_{S}^{0}, B^{0} / \bar{B}^{0} \rightarrow \psi(2 S) K_{S}^{0}$ and $B^{0} / \bar{B}^{0} \rightarrow J / \psi K_{L}^{0}$ events, as described in detail in ${ }^{6}$.

The selection of the two modes with the $K_{S}^{0}$ can profit from the fact that there are two discriminating kinematic variables, $\Delta E$, the difference between the reconstructed and expected 

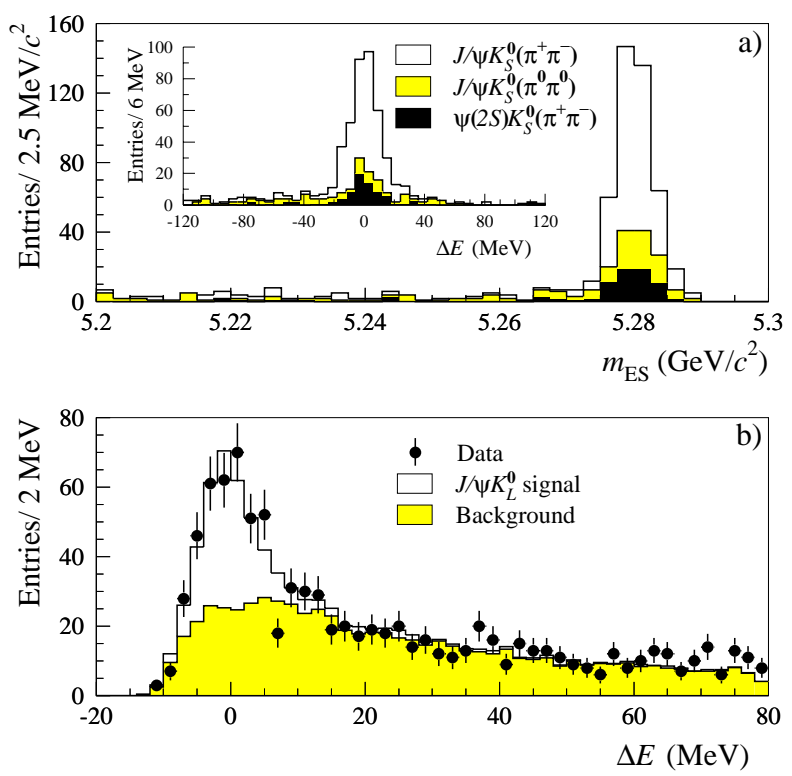

Figure 1: $J / \psi K_{S}^{0}\left(K_{S}^{0} \rightarrow \pi^{+} \pi^{-}, \pi^{0} \pi^{0}\right)$ and $\psi K_{S}^{0}\left(K_{S}^{0} \rightarrow \pi^{0} \pi^{0}\right)$ signal (top) and $J / \psi K_{L}^{0}$ signal (bottom).

$B$ meson energy measured in the center-of-mass frame, and $m_{\mathrm{ES}}$, the beam-energy substituted mass (see Fig. 1). The background is mainly coming from combinatorics in continuum and other $B$ decays and its properties can be estimated from the $m_{\mathrm{ES}}$ sidebands.

The $J / \psi K_{L}^{0}$ sample is instead less pure because the the $K_{L}^{0}$ momentum is not reconstructed. The $B$ mass constraint is therefore imposed and only one discriminating variable is left ( $\Delta E$ ) as shown in Fig. 1. The background is mainly due to other $B \rightarrow J / \psi X$ decays and its properties are determined on MC.

Signal event yields and purities, determined from a fit to the $m_{\mathrm{ES}}$ distributions after selection on $\Delta E$, are summarized in Table 1.

- Selection of decays in flavor eigenstate $\left(B_{\text {flav }}\right) . B^{0}$ candidates are formed by combining a $D^{*}$ or $D^{+}$with a $\pi^{+}, \rho^{+}\left(\rho^{+} \rightarrow \pi^{+} \pi^{0}\right), a_{1}^{+}\left(a_{1}^{+} \rightarrow \pi^{+} \pi^{-} \pi^{+}\right)$, or by combining a $J / \psi$ candidate with a $K^{* 0}\left(K^{* 0} \rightarrow K^{-} \pi^{+}\right)^{5}$. Their background is mainly due to conbinatorics and can be studied in the $m_{\mathrm{ES}}$ sidebands. Yields and purities are also summarized in Table 1.

- Measurement of the distance $\Delta z$ between the vertex of the reconstructed $B$ meson $\left(B_{\text {rec }}\right)$ and the vertex of the flavor-tagging $B$ meson $\left(B_{\mathrm{tag}}\right)$.

In the reconstruction of the $B_{\text {rec }}$ vertex, we use all charged daughter tracks. The vertex for the $B_{\text {tag }}$ decay is constructed from all the remaining tracks in the event.

In order to reduce bias and tails due to long-lived particles, $K_{S}^{0}$ and $\Lambda^{0}$ candidates are used as input to the fit in place of their daughters. In addition, tracks consistent with photon conversions $\left(\gamma \rightarrow e^{+} e^{-}\right)$are excluded. To reduce contributions from charm decay products, which bias the determination of the vertex position, the track with the largest vertex $\chi^{2}$ contribution greater than 6 is removed and the fit is redone until no track fails the $\chi^{2}$ requirement or only one track remains. 
Table 1: Number of tagged events, signal purity and result of fitting for $C P$ asymmetries in the full $C P$ sample and in various subsamples, as well as in the $B_{\text {flav }}$ and charged $B$ control samples.

\begin{tabular}{|c|c|c|c|}
\hline Sample & $N_{\text {tag }}$ & Purity (\%) & $\sin 2 \beta$ \\
\hline \hline$J / \psi K_{S}^{0}, \psi(2 S) K_{S}^{0}$ & 273 & $96 \pm 1$ & $0.25 \pm 0.22$ \\
$J / \psi K_{L}^{0}$ & 256 & $39 \pm 6$ & $0.87 \pm 0.51$ \\
\hline Full $C P$ sample & 529 & $69 \pm 2$ & $0.34 \pm 0.20$ \\
\hline \hline$J / \psi K_{S}^{0}, \psi(2 S) K_{S}^{0}$ only & & & \\
\hline$J / \psi K_{S}^{0}\left(K_{S}^{0} \rightarrow \pi^{+} \pi^{-}\right)$ & 188 & $98 \pm 1$ & $0.25 \pm 0.26$ \\
$J / \psi K_{S}^{0}\left(K_{S}^{0} \rightarrow \pi^{0} \pi^{0}\right)$ & 41 & $85 \pm 6$ & $-0.05 \pm 0.66$ \\
$\psi(2 S) K_{S}^{0}\left(K_{S}^{0} \rightarrow \pi^{+} \pi^{-}\right)$ & 44 & $97 \pm 3$ & $0.40 \pm 0.50$ \\
\hline Lepton tags & 34 & $99 \pm 2$ & $0.07 \pm 0.43$ \\
Kaon tags & 156 & $96 \pm 2$ & $0.40 \pm 0.29$ \\
NT1 tags & 28 & $97 \pm 3$ & $-0.03 \pm 0.67$ \\
NT2 tags & 55 & $96 \pm 3$ & $0.09 \pm 0.76$ \\
\hline \hline$B_{\text {flav } \text { sample }}$ & 4637 & $86 \pm 1$ & $0.03 \pm 0.05$ \\
\hline Charged $B$ sample & 5165 & $90 \pm 1$ & $0.02 \pm 0.05$ \\
\hline
\end{tabular}

From the measurement of $\Delta z$ and of the $B$ momentum, $\Delta t$ can be computed. At an asymmetric-energy $B$ Factory, in fact, the proper decay-time difference $\Delta t$ is, to an excellent approximation, proportional to the distance $\Delta z$ between the two $B^{0}$-decay vertices along the axis of the boost, $\Delta t \approx \Delta z / \mathrm{c}\langle\beta \gamma\rangle$.

The time resolution function in equation 2 is described by a sum of three Gaussian distributions (called the core, tail and outlier components) with different means and widths:

$$
\mathcal{R}\left(\delta_{\mathrm{t}} ; \hat{a}\right)=\sum_{k=1}^{2} \frac{f_{k}}{\sigma_{k} \sqrt{2 \pi}} \exp \left(-\frac{\left(\delta_{\mathrm{t}}-\delta_{k}\right)^{2}}{2 \sigma_{k}^{2}}\right)+\frac{f_{3}}{\sigma_{3} \sqrt{2 \pi}} \exp \left(-\frac{\delta_{\mathrm{t}}^{2}}{2 \sigma_{3}^{2}}\right) .
$$

For the core and tail Gaussians, the widths are scaled by the event-by-event measurement error $\sigma_{\Delta t}$ derived from the vertex fits: $\sigma_{1,2}=\mathcal{S}_{1,2} \times \sigma_{\Delta t}$. In data, approximately $65 \%$ of the area of the resolution function is in the core Gaussian. The width of the core Gaussian is approximately $110 \mu \mathrm{m}$ or $0.7 \mathrm{ps}$; the width of the tail Gaussian is approximately $300 \mu \mathrm{m}$ or $1.8 \mathrm{ps}$. The third Gaussian has a fixed width of $8 \mathrm{ps}$ and no offset; it accounts for the fewer than $1 \%$ of events with incorrectly reconstructed vertices.

- Determination of the flavor of the $B_{t a g}$.

Each event with a $C P$ candidate is assigned a $B^{0}$ or $\bar{B}^{0}$ tag if the rest of the event (i.e., with the daughter tracks of the $B_{C P}$ removed) satisfies the criteria from one of several tagging categories. In other words, a $B^{0}$ tag indicates that the $B_{C P}$ candidate was in a $\bar{B}^{0}$ state at $\Delta t=0 ;$ a $\bar{B}^{0}$ tag indicates that the $B_{C P}$ candidate was in a $B^{0}$ state.

Two tagging categories rely on the presence of a fast lepton (Lepton category) and/or one or more charged kaons in the event (Kaon category). Two categories, called neural network categories (NT1 and NT2), are based upon the output value of a neural network algorithm applied to events that have not already been assigned to lepton or kaon tagging categories. 
Table 2: Mistag fractions measured from a maximum-likelihood fit to the time distribution for the fully-reconstructed $B^{0}$ sample. The uncertainties on $\varepsilon$ and $Q$ are statistical only.

\begin{tabular}{|c|c|c|c|c|}
\hline Category & $\varepsilon(\%)$ & $w(\%)$ & $\Delta w(\%)$ & $Q(\%)$ \\
\hline \hline Lepton & $10.9 \pm 0.4$ & $11.6 \pm 2.0$ & $3.1 \pm 3.1$ & $6.4 \pm 0.7$ \\
Kaon & $36.5 \pm 0.7$ & $17.1 \pm 1.3$ & $-1.9 \pm 1.9$ & $15.8 \pm 1.3$ \\
NT1 & $7.7 \pm 0.4$ & $21.2 \pm 2.9$ & $7.8 \pm 4.2$ & $2.6 \pm 0.5$ \\
NT2 & $13.7 \pm 0.5$ & $31.7 \pm 2.6$ & $-4.7 \pm 3.5$ & $1.8 \pm 0.5$ \\
\hline \hline All & $68.9 \pm 1.0$ & & & $26.7 \pm 1.6$ \\
\hline
\end{tabular}

The figure of merit for each tagging category is the effective tagging efficiency $Q_{i}=\varepsilon_{i}\left(1-2 w_{i}\right)^{2}$, where $\varepsilon_{i}$ is the fraction of events assigned to category $i$ and $w_{i}$ is the mistag fraction. The effective tagging efficiency as evaluated in data is summarized in Tab. 4.1.

- The mistag fractions and the tagging efficiencies obtained by combining the results from maximum likelihood fits to the time distributions in the $B^{0}$ hadronic and semileptonic samples are summarized in Table 4.1.

- Extraction of the amplitude of the $C P$ asymmetry and the value of $\sin 2 \beta$ with an unbinned maximum likelihood fit. In order to extract as much information from the data itself and properly account for correlation, the fit is performed simultaneously to the $C P$ and the flavor eigenstates. There are 35 parameters free in the fit:

\section{- Value of $\sin 2 \beta$;}

- Signal resolution function: Nine parameters $\hat{a}_{i}$ to describe the resolution function for the signal, being scale factors $S_{1,2}$ for the event-by-event $\Delta z$ resolution errors of the core and tail Gaussian components, individual core biases $\delta_{1, i}$ per tagging category and a common tail bias $\delta_{2}$, and the tail $f_{1}$ and outlier $f_{3}$ fractions; the width of the outlier component is taken to be a fixed 8 ps with zero bias;

- Signal dilutions: Eight parameters to describe the measured average dilutions $\left\langle\mathcal{D}_{i}\right\rangle$ and dilution differences $\delta \mathcal{D}_{i}$ in each tagging category.

- Background resolution function: Three parameters are used to describe a common resolution function for all non-peaking backgrounds, which is taken as a single Gaussian distribution with a scale factor $S_{1}$ for the event-by-event $\Delta z$ errors and an common bias $\delta_{1}$, and an outlier fraction $f_{3}$; the width of the outlier component is taken to be a fixed 8 ps with zero bias;

- $B_{\text {flav }}$ background properties: A total of 13 parameters describe the $B_{\text {flav }}$ background properties. We make several assumptions to simplify the parameterization of the background contributions and assign a corresponding systematic uncertainty. The mixing background contribution is assumed to be absent, $f_{i, 3}^{\text {flav }}=0$. The size of the peaking background is determined from Monte Carlo simulation to be $\delta_{\text {peak }}^{\text {flav }}=1.5 \pm 0.5 \%$ of the signal contribution in each tagging category. This contribution is dominantely from $\mathrm{B}^{+}$ events, so $\Delta m_{d}=0, \Gamma_{i, \text { peak }}^{\text {flav }}=\Gamma_{B^{+}}$and $D_{i, \text { peak }}^{\text {flav }}$ are taken from the $B^{+}$data sample. The effective dilutions for the prompt $\left(D_{i, 1}^{\text {flav }}, 4\right.$ parameters $)$ and lifetime $\left(D_{i, 2}^{\text {flav }}, 4\right.$ parameters) contributions are allowed to vary. The relative amount of these two contributions 

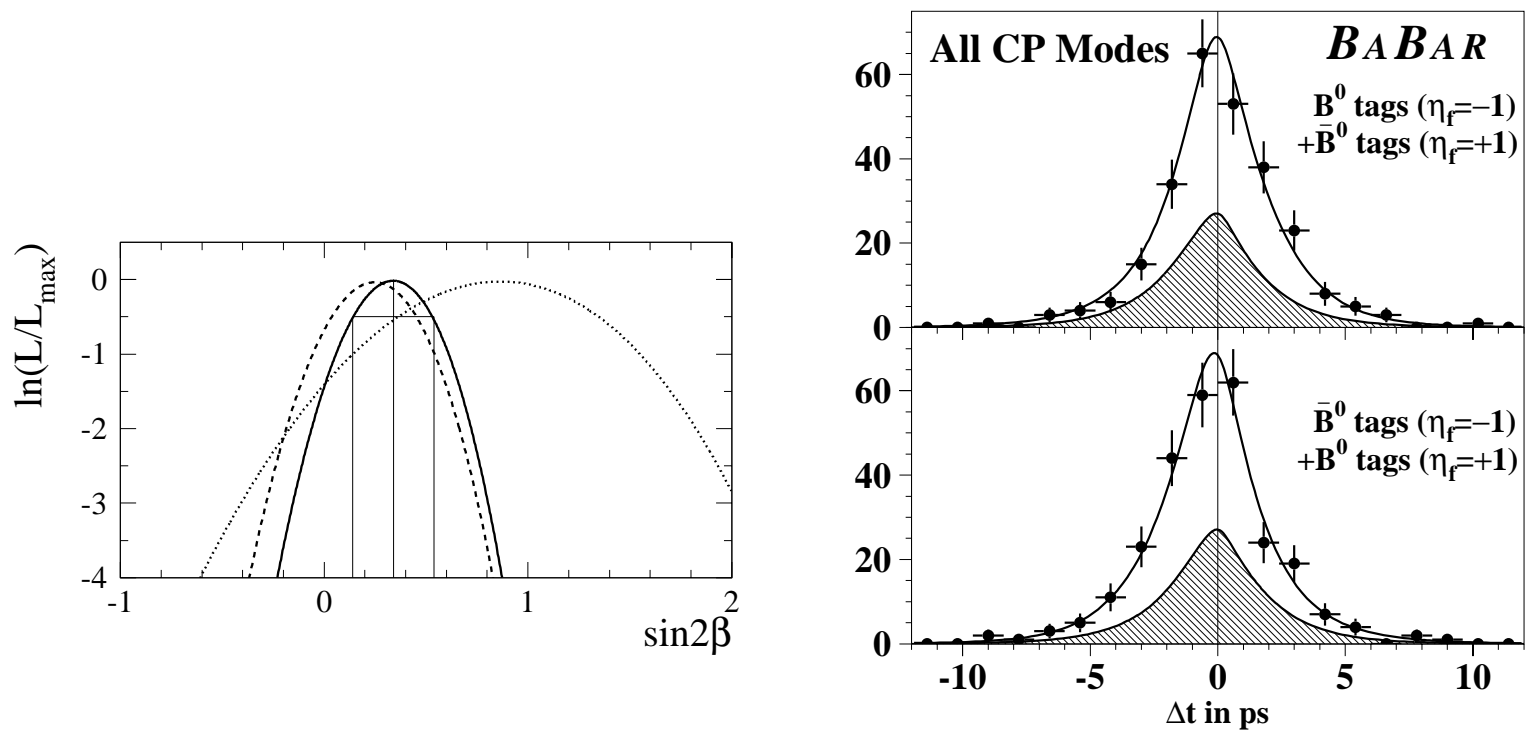

Figure 2: Variation of the log likelihood as a function of $\sin 2 \beta$ (left), for the whole sample (full curve), the $\eta_{C P}=-1$ sample (dashed curve) and the $\eta_{C P}=1$ sample (dotted line). Distribution of $\Delta t$ for $(a)$ the $B^{0}$ tagged events and $(b)$ the $\bar{B}^{0}$ tagged events in the CP sample (right).

is allowed to vary, independently in each tagging category (4 parameters). For the lifetime contribution, $\Gamma_{i, 2}^{\text {flav }}$ is assumed to be same for all tagging categories, giving one free parameter.

- $C P$ background properties: One parameter, the fraction of prompt relative to lifetime background, assumed to be the same for each tagging category, is allowed to float to describe the $C P$ background properties. The effective dilutions of the lifetime and peaking contribution are set to zero $\left(D_{i, 2}^{C P}=D_{i \text {,peak }}^{C P}=0\right)$, corresponding to no $C P$ asymmetry in the background. The size and parameters of the peaking background is again determined from Monte Carlo simulation. The fraction of peaking background is $\delta_{\text {peak }}^{C P}=1 \pm 1 \%$ of the signal contribution, independent of tagging category. This contribution is assumed to have dilutions and lifetime parameters in common with the signal contribution. Finally, the lifetime of the lifetime background is assumed to be $\tau_{B^{0}}$ in all tagging categories.

The maximum-likelihood fit for $\sin 2 \beta$, using the full tagged sample, gives:

$$
\sin 2 \beta=0.34 \pm 0.20 \text { (stat) } \pm 0.05 \text { (syst) }
$$

For this result, the $B^{0}$ lifetime and $\Delta m_{d}$ are fixed to the current best values ${ }^{7}$. The $\log$ likelihood is shown as a function of $\sin 2 \beta$ and the $\Delta t$ distributions for $B^{0}$ and $\bar{B}^{0}$ tags are shown in Fig. 2.

The dominant sources of systematic error are the assumed parameterization of the $\Delta t$ resolution function (0.04), due in part to residual uncertainties in the SVT alignment, and uncertainties in the level, composition, and $C P$ asymmetry of the background in the selected $C P$ events (0.02). The systematic errors from uncertainties in $\Delta m_{B^{0}}$ and $\tau_{B^{0}}$ and from the parameterization of the 
background in the selected $B_{\text {flav }}$ sample are found to be negligible. An increase of $0.02 \hbar \mathrm{ps}^{-1}$ in the assumed value for $\Delta m_{B^{0}}$ decreases $\sin 2 \beta$ by 0.012 .

The large sample of reconstructed events allows a number of consistency checks, including separation of the data by decay mode, tagging category and $B_{\text {tag }}$ flavor. The results of fits to these subsamples are shown in Table 1 for the high-purity $K_{S}^{0}$ events. Table 1 also shows results of fits with the samples of non- $C P$ decay modes, where no statistically significant asymmetry is found.

\section{Measurements of charged and neutral $B$ meson lifetimes and $B^{0} \bar{B}^{0}$ oscillations}

These measurements can be used to test theoretical models of heavy-quark decays and to constrain the Unitarity Triangle (via the sensitivity to the value of the CKM matrix element $V_{t d}$ ).

One $B\left(B_{r e c}\right)$ is fully reconstructed in an all-hadronic $\left(B^{0} \rightarrow D^{(*)-} \pi^{+}, D^{(*)-} \rho^{+}, D^{(*)-} a_{1}^{+}\right.$, $J / \psi K^{* 0}$ and $\left.B^{+} \rightarrow \bar{D}^{(*) 0} \pi^{+}, J / \psi K^{+}, \psi(2 S) K^{+}\right)$modes. The number of selected events and purities are summarized in Table 1.

The measurement of $\Delta t$ and, when needed, the tagging of the recoiling $B$ is done in the same way as for the $\sin 2 \beta$ measurement, so that these measurements constitute also a valuable validation of the $C P$ measurement.

\subsection{Lifetime Measurements}

The $B^{0}$ and $B^{+}$lifetimes are extracted from a simultaneous unbinned maximum likelihood fit to the $\Delta t$ distributions of the signal candidates, assuming a common resolution function. An empirical description of the $\Delta t$ background shape is assumed, using $m_{\mathrm{ES}}$ sidebands with independent parameters for neutral and charged mesons. Fig. 3 shows the $\Delta t$ distributions with the fit result superimposed.
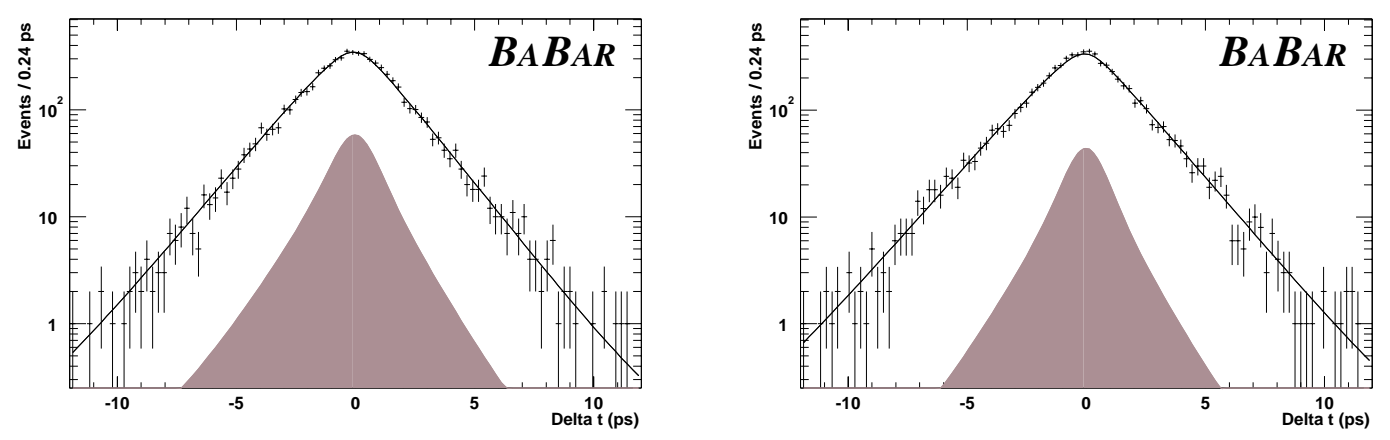

Figure 3: $\Delta t$ distributions for $B^{0} / \bar{B}^{0}$ (left) and $B^{+} / B^{-}$(right) candidates in the signal region $\left(m_{\mathrm{ES}}>5.27 \mathrm{GeV} / c^{2}\right)$. The result of the lifetime fit is superimposed. The background is shown by the hatched area.

\subsection{Time-dependent $B^{0} \bar{B}^{0}$ mixing}

A time-dependent $B^{0} \bar{B}^{0}$ mixing measurement requires the determination of the flavor of both $B$ mesons. Considering the $B^{0} \bar{B}^{0}$ system as a whole, one can classify the tagged events as mixed or unmixed depending on whether the $B_{t a g}$ is tagged with the same flavor as the $B_{r e c}$ or with the opposite flavor. 
From the time-dependent rate of mixed $\left(N_{\text {mix }}\right)$ and unmixed $\left(N_{\text {unmix }}\right)$ events, the mixing asymmetry $a(\Delta t)=\left(N_{\text {unmix }}-N_{\text {mix }}\right) /\left(N_{\text {unmix }}+N_{\text {mix }}\right)$ is calculated as a function of $\Delta t$ and fit to the expected cosine distribution. A likelihood fit with 34 free parameters is performed. The free parameters are the same as in the $\sin 2 \beta$ fit, apart from $\sin 2 \beta$ itself and the background to the $C P$ eigenstates which are not used, and $\Delta m_{d}$, which is, of course, floated.
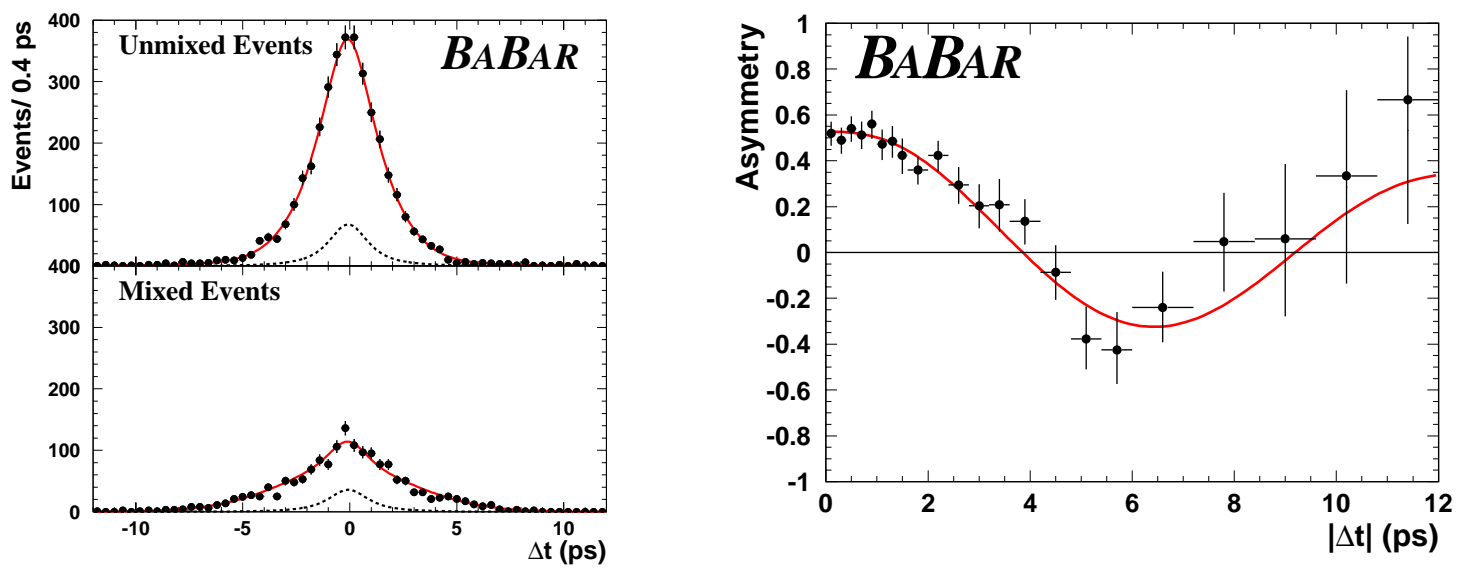

Figure 4: $\Delta t$ distribution for mixed and unmixed events (left) and time-dependent asymmetry a $(\Delta t)$ between unmixed and mixed events (rigth)

Fig. 4 shows the $\Delta t$ and $a(\Delta t)=\left(N_{\text {unmix }}-N_{\text {mix }}\right) /\left(N_{\text {unmix }}+N_{\text {mix }}\right)$ distributions with the fit result superimposed.

\subsection{Results}

The preliminary results for the $B$ meson lifetimes are $\tau_{B^{0}}=1.546 \pm 0.032$ (stat) \pm 0.022 (syst) ps and $\tau_{B^{+}}=1.673 \pm 0.032$ (stat) \pm 0.022 (syst) ps and for their ratio is $\tau_{B^{+}} / \tau_{B^{0}}=1.082 \pm 0.026$ (stat) \pm 0.011 (syst).

We measure the $B^{0} \bar{B}^{0}$ oscillation frequency: $\Delta m_{d}=0.519 \pm 0.020$ (stat) \pm 0.016 (syst) $\hbar \mathrm{ps}^{-1}$

The above results are consistent with previous measurements ${ }^{7}$ and are of similar precision. The mixing result is compatible with a $B A B A R$ measurement using di-leptons ${ }^{9}$.

\section{Conclusions}

We have presented $B A B A R$ 's first measurement of the $C P$-violating asymmetry parameter $\sin 2 \beta$ in the $B$ meson system:

$$
\sin 2 \beta=0.34 \pm 0.20 \text { (stat) } \pm 0.05 \text { (syst) }
$$

Our measurement is consistent with the world average $\sin 2 \beta=0.9 \pm 0.4^{7}$, and is currently limited by the size of the $C P$ sample.

We have also presented time-dependent mixing and lifetime measurements, performed for the first time at the $\Upsilon(4 S)$.

\section{References}

1. P. H. Harrison and H. R. Quinn, eds., "The BABAR physics book", SLAC-R-504 (1998). 
2. BABAR Collaboration, B. Aubert et al., "The first year of the BABAR experiment at PEP-II", hep-ex/0008059.

3. BABAR Collaboration, B. Aubert et al., "The BABAR Detector" hep-ex/0105044, to appear in to Nucl. Instr. and Methods .

4. BABAR Collaboration, B. Aubert et al., "Measurement of $C P$ violating asymmetries in $B^{0}$ decays to CP eigenstates", Phys.Rev.Lett. 86, 2515 (2001).

5. BABAR Collaboration, B. Aubert et al., "A measurement of the $B^{0} \bar{B}^{0}$ oscillation frequency and determination of flavor-tagging efficiency using semileptonic and hadronic $B$ decays", hep-ex/0008052.

6. BABAR Collaboration, B. Aubert et al., "Exclusive $B$ decays to charmonium final states", hep-ex/0008050.

7. Particle Data Group, D. E. Groom et al., Eur. Phys. Jour. C 15, 1 (2000).

8. BABAR Collaboration, B. Aubert et al. "A study of time-dependent $C P$-asymmetries in $B^{0} \rightarrow J / \psi K_{S}^{0}$ and $B^{0} \rightarrow \psi(2 S) K_{S}^{0}$ decays", hep-ex/0008048.

9. BABAR Collaboration, B. Aubert et al., "Measurement of the time dependence of $B^{0} \bar{B}^{0}$ oscillations using inclusive dilepton events", hep-ex/0008054. 